\title{
Human Supervised Multirotor UAV System Design for Inspection Applications
}

\author{
Mohammad Shaqura \\ Mechanical Engineering \\ King Abdullah University \\ of Science and Technology
}

Thuwal, KSA

\author{
Khalid Alzuhair \\ Electrical Engineering \\ King Abdullah University \\ of Science and Technology \\ Thuwal, KSA
}

\author{
Fadl Abdellatif \\ Intelligent Systems \\ $R \& D$ Center \\ Saudi Aramco \\ Thuwal, KSA
}

\author{
Jeff S. Shamma \\ Electrical Engineering \\ King Abdullah University \\ of Science and Technology \\ Thuwal, KSA
}

\begin{abstract}
Multirotor UAVs are widely used for aerial inspection applications where missions are accomplished either via manual or autonomous control. Human controlled UAVs require trained pilots which can be a barrier from using the technology for general inspection personnel. Fully autonomous navigation, which employs onboard sensing, planning and coverage algorithms, is effective but comes with the cost of development and operational complexities. A human supervised UAV system design is presented where a deployed aerial vehicle operates in semi-autonomous mode. An operator, who is equipped with a smart handheld laser pointer, gives the UAV global guiding directions to reach the inspection target. The UAV is equipped with onboard vision sensing for local planning and target identification in addition to video streaming or recording. System operation is validated in indoor flight tests.

Index Terms-Unmanned aerial vehicles, Inspection, Autonomous systems, Human-robot interaction
\end{abstract}

\section{INTRODUCTION}

Multirotors are popular aerial robotics platforms due to their high maneuverability and mobility. Unlike conventional fixed-wing UAVs, their high agility and unconstrained translational velocity make them suitable for multiple applications that require autonomous or semi-autonomous operations. They are integrated into applications where a higher level of mission efficiency is needed or in applications where human access is limited. Remotely controlled and autonomous UAVs are widely used for search and rescue and disaster assessment similar to [2]. Lower cost and less complex environmental sensing is enabled by the advancement of UAV technology [3].

Multirotor UAVs are deployed in several UAV applications where efficiency and accuracy are desired. Technological advancements have increased robustness and durabilities of these systems. They can also be equipped with state-of-theart sensing and communication devices that are essential for remote inspection applications. Authors in [4], proposed a method for high infrastructure inspection using quadcopter UAVs. A system for asphalt pavement inspection using aerial robots is presented in [5]. Power lines and wall inspection using quadcopters are presented in [7] and [6] respectively.

For some applications, full autonomy of systems is an advantage. However, for many practical systems, human

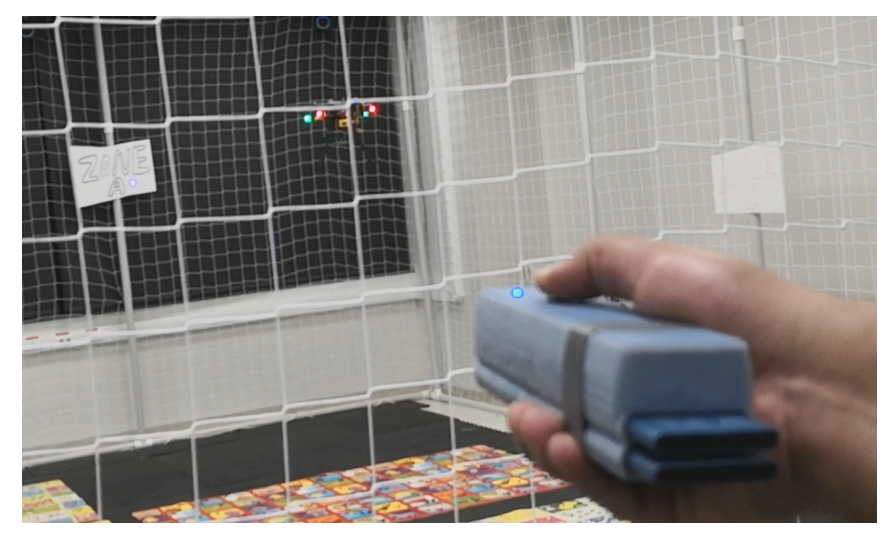

Fig. 1. Human supervised aerial system indoor testing

supervisory can greatly enhance efficiency and reduce complexity. Semi-autonomous systems involve human-robot interactions where control actions are co-decisions taken by operators and machines. An approach that decodes human gestures to control quadcopter UAVs is presented in [8]. Similarly, authors in [9] proposed a system that employs stereo cameras and depth maps to convert gestures to control signals sent to drones. A system that uses mental signals and eye movement to control a quadcopter is developed in [10] where an operator wears an EEG headset with eye tracking camera as the sensing devices. Fixed laser guidance system for ground robots are presented in [16] where a fixed position laser source is used to guide multiple ground robots that are equipped with laser sensing and communication.

The objective of the proposed aerial inspection system is to eliminate the need for advanced pilot skills from a standard worker. A semi-autonomous aerial inspection system design and implementation are presented in this work. The system consists of two main components: 1) A quadcopter UAV with onboard sensing and computation. 2) A smart handheld pointer with embedded sensing and communication. The Robot Operating System, $(R O S)$ is utilized to connect the system's components and implement the control algorithms [1]. The pointing device is used by an operator to point at an inspection target. The relative direction vector guides a quad- 

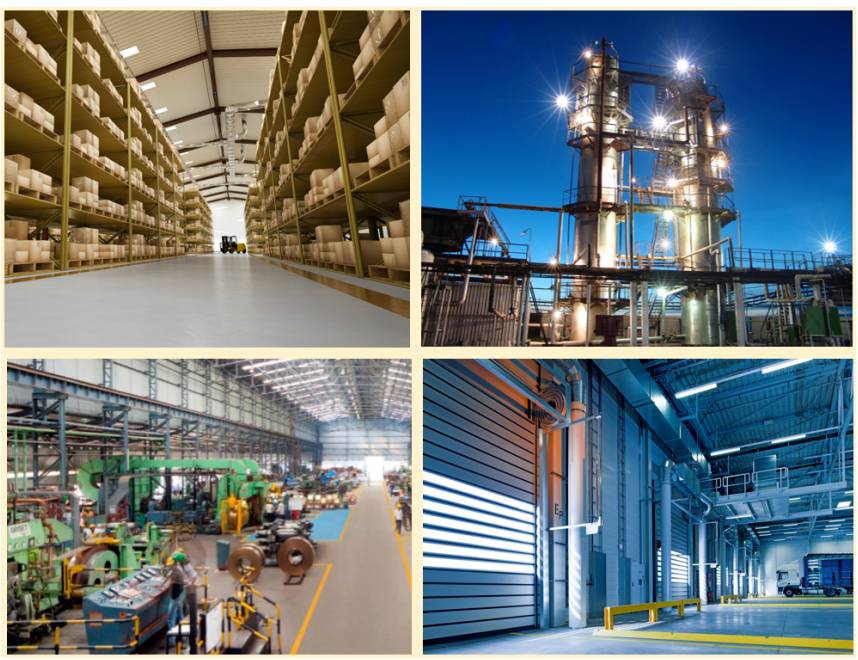

Fig. 2. Examples of different inspection applications

copter UAV to navigate to the target. Once it is in the target's neighborhood, the UAV utilizes its onboard vision sensing to align itself for best subject visibility. This article is organized as follows: section II presents the problem definitions and environment specifications. Supervised navigation approach is presented in section III. Then, the proposed system design and implementation are presented in section IV. Experimental validation in indoor flight tests are presented in sections $\mathrm{V}$ before discussing future directions of the project.

\section{Problem Definition and Current Systems}

Inspection problem definition along with the current systems' configuration for aerial inspection and proposed system are discussed in this section.

\section{A. Environment Specifications}

The scope of this work is the inspection in indoor or confined outdoor environments. We assume relatively static features during an inspection task. The size of the UAV can be scaled to fit into the target environment. Examples of such environments are shown in fig. 2. The inspection target is usually within line of sight from the operator but far from bare eyes inspection. Visibility of targets depends on intermediate obstacles in the environment, operator vision and ambient conditions. Environments can vary from well structured patterned places, e.g. warehouses to more challenging unusual environments, e.g. fabrication workshops or earthquake sites.

\section{B. Inspection Mission}

Inspection missions can be categorized into two main types, periodic inspection, and on-demand inspection. Industrial facilities often have for instance annual inspection plans in place for quality control and early fix of potential problems. Inspection using aerial robots has become popular in the recent years motivated by the advancement of technology and the lower cost and higher efficiency compared to classical techniques, for instance, scaffolding. Data produced by aerial inspection systems usually comes in the form of still images or video streams that help the operator to assess situations and take actions accordingly. Some systems are developed for specific applications where computer vision algorithms are employed for automated assessment.

\section{Configuration of Current Systems}

Here we present the two main common configurations in currently deployed inspection systems. Comprehensive surveying of all available technologies is outside the scope of this paper. Related surveys can be found in [11] and [12].

1) Autonomous UAV Systems: In an autonomous system setup, the UAV is programmed to execute certain navigation objective that achieves inspection goals. This can be in the form of optimal coverage, sense and avoid or preplanned path. A typical aerial autonomous system is shown in fig. 3(a). Such systems are typically equipped with advanced sensing, e.g., lidars, stereo systems, and the inertial and navigation sensors.

2) Remotely Piloted Systems: Operator controlled aerial inspection is more common where the UAV is controlled by a trained operator who controls the vehicle using a joystick, see fig. 3(b). The flight can be assisted where the onboard flight controller takes care of certain low-level aspects related to stability and state limits. First-person view (FPV) enables pilots to navigate to areas where an operator line-of-sight is not granted. Operating such systems need a considerable amount of training and a certain level of experience. Often, the inspection mission is executed by a third party specialist.

\section{Proposed System}

The proposed system (fig. 3(c)) aims to bridge the gap between autonomy and human supervisory, mainly focusing on ease of operation and minimal training. The system consists of a smart hand-held laser pointer with embedded micro-controller, 9-axis inertial measurement unit (IMU) and Wifi antenna. The device communicates with the UAV companion computer via Wifi. Operators use the laser to get self-reference of the inspection target. The direction vector from the UAV to the target is calculated using the pointer's IMU measurements and the relative initial pose of the UAV with respect to the operator. After that, the UAV navigates along the generated guiding vector, using its onboard sensing and control, until it is in the neighborhood of the target. Once the laser light is detected by the onboard camera, vision feedback is utilized to adjust the position of the vehicle for optimal frame coverage.

\section{QUADCOPTER NAVIGATION APPROACH}

Three coordinate frames are defined in this scope, an inertial reference frame, a UAV body frame, and a pointing device frame as shown in figure 4. The inertial frame $\left(x_{a}, y_{a}, z_{a}\right)$ is a fixed global reference. The UAV body frame $\left(x_{q}, y_{q}, z_{q}\right)$ follows the convention of the onboard flight controller. The frame of the pointing device $\left(x_{p}, y_{p}, z_{p}\right)$ refers to the embedded IMU axes. We assume the human operator can point to targets in 3D space moving the pointer around $y_{p}$ (pitch, $\theta_{p}$ ) and $z_{p}\left(\right.$ yaw, $\left.\psi_{p}\right)$ axes. 


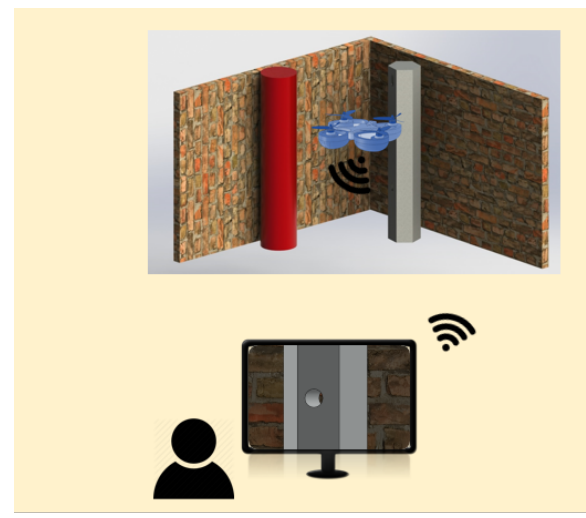

(a)
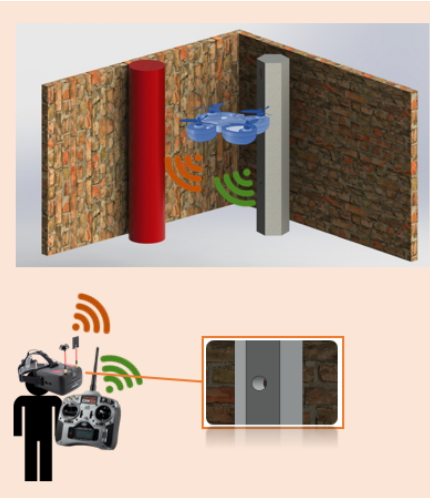

(b)

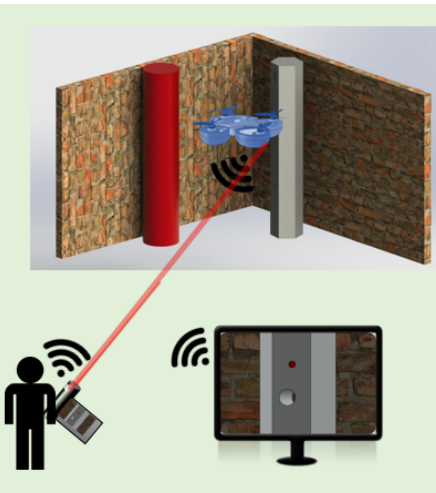

(c)

Fig. 3. Different aerial inspection systems configurations: (a) fully autonomous system, no operator interaction with control. (b) Manually controlled fpv system, operator control UAV using joystick and receive imagery feedback on wearable headset. (c) Operator guides UAV to reach inspection target, on-board UAV sensing and control are used for local planning

During system initialization, the operator is assumed to be static until receiving LED ready flag that is sent from the UAV to the pointing device. The operator remains inside a control base throughout the mission. In situations where an operator moves freely in the inspection space, IMU data fusion can be utilized for pose and speed estimation of the operator similar to [13] and [14], to update the reference position of the operator with respect to the flying vehicle.

The initial pitch angles of the pointer and the UAV are assumed to be sufficiently small, $\theta_{p_{i}} \sim 0, \theta_{q_{i}} \sim 0$, their initial headings are normally different. This difference, referred to as the heading offset, is given by:

$$
\Delta \psi_{i}=\psi_{p_{i}}-\psi_{q_{i}}
$$

The guidance algorithm, illustrated in fig. 4, calculates a set of waypoints, that are separated by a step size $s$, which guide the UAV along a path closely following the laser line. The waypoints do not exactly coincide with the laser line but are rather offset from it by a distance $r$ to prevent the UAV from obstructing the laser which would otherwise make it difficult for the operator to point at the desired target. In order to facilitate the waypoint calculation, five 3-dimensional vectors are defined. The first two are defined in the inertial global frame $\left(x_{a}, y_{a}, z_{a}\right)$ and they are the pointer position (operator position) $\vec{P}$ and the UAV position $\vec{Q}$ in 3D space as follows:

$$
\vec{P}=\left[\begin{array}{l}
p_{x} \\
p_{y} \\
p_{z}
\end{array}\right] \quad, \quad \vec{Q}=\left[\begin{array}{l}
q_{x} \\
q_{y} \\
q_{z}
\end{array}\right]
$$

The laser unit vector $\vec{L}$ is defined in the frame of the pointing device $\left(x_{p}, y_{p}, z_{p}\right)$ and it has a length of one unit. It always points along the laser line towards the target and is calculated using the orientation measurements obtained from the embedded IMU in the pointer as follows:

$$
\vec{L}=\left[\begin{array}{c}
l_{x} \\
l_{y} \\
l_{z}
\end{array}\right]=\left[\begin{array}{c}
\cos \left(\theta_{p}\right) \cos \left(\psi_{p}\right) \\
\cos \left(\theta_{p}\right) \sin \left(\psi_{p}\right) \\
\sin \left(\theta_{p}\right)
\end{array}\right]
$$

In order to calculate the first waypoint, the projection of the UAV position onto the laser line must first be obtained which defines the projected position vector $\vec{N}$ in the pointing device reference frame $\left(x_{p}, y_{p}, z_{p}\right)$ as follows:

$$
\vec{N}=\left[\begin{array}{l}
n_{x} \\
n_{y} \\
n_{z}
\end{array}\right]=(\vec{L} \cdot(\vec{Q}-\vec{P})) \vec{L}
$$

The laser occlusion offset $r$ is applied to calculate the position vector of the first waypoint $\vec{W}$ defined as well in the pointing device reference frame as follows:

$$
\vec{W}=\left[\begin{array}{l}
w_{x} \\
w_{y} \\
w_{z}
\end{array}\right]=\vec{N}+r \frac{\vec{Q}-\vec{P}-\vec{N}}{\|\vec{Q}-\vec{P}-\vec{N}\|}
$$

Each consecutive waypoint is given by the following equation which incorporates a simple addition of predefined steps parallel to the laser line.

$$
\vec{W}_{k}=\vec{W}+k s \vec{L}
$$

The waypoints are converted to the global inertial frame $\left(x_{a}, y_{a}, z_{a}\right)$, as shown in the following equation, which are fed to the UAV at a constant frequency to achieve the desired speed until the target or an obstacle has been detected.

$$
\vec{W}_{k \text { global }}=\vec{W}_{k}+\vec{P}
$$

While waypoints are used to control the position of the $\mathrm{UAV}$, its heading command $\psi_{c}$ is set such that it is pointing toward the target while compensating for any initial deviation between the UAV heading and that of the pointer.

$$
\psi_{c}=\psi_{q}+\psi_{p}-\Delta \psi_{i}
$$

Once the drone is in the neighborhood of the target, a vision based control mode is activated if the laser dot is detected by the camera. The switch to vision-based control is triggered by the UAV onboard sensing. The UAV stays around hover state and starts adjusting its position in the body frame $\left(x_{q}, y_{q}, z_{q}\right)$ in order to align the camera with 
the desired view. In the inertial frame, the position vector is represented by:

$$
\left[\begin{array}{l}
x_{a} \\
y_{a} \\
z_{a}
\end{array}\right]=\left[\begin{array}{ccc}
\cos \left(\psi_{q}\right) & -\sin \left(\psi_{q}\right) & 0 \\
\sin \left(\psi_{q}\right) & \cos \left(\psi_{q}\right) & 0 \\
0 & 0 & 1
\end{array}\right]\left[\begin{array}{l}
x_{q} \\
y_{q} \\
z_{q}
\end{array}\right]
$$

During the vision based alignment control, the vehicle remains in near hover state with $\theta_{q} \sim 0$ and $\phi_{q} \sim 0$. The UAV is assumed to have onboard obstacle avoidance sensing and mechanism, such as stereo vision or ultrasound. When no pointing command is given, the UAV returns to its home position and hovers in place until receiving the next inspection command from the operator.

Visual Laser Light Sensing: the vision algorithm used consists of three stages as shown in figure 5. For detecting the laser light, the camera is configured to capture with low exposure settings. This reduces reflections and focuses the image on shiny elements. Exposure is experimentally adjusted based on the used camera as an exposure that is too low can confuse laser reflections with other light sources in the environment. The second stage is applying an RGB filter to the image using a range that differentiates the purple laser reflection from surfaces with different colors and finishes. The resulting image includes the laser dot and some light reflection noise depending on the target orientation. A Hough circle transform is applied to the image to search for and detect the correct circle [15]. The radius range of the circle formed by the laser reflection can be computed offline and mapped to a lookup table that is a function of the estimated distance between the source and the target.

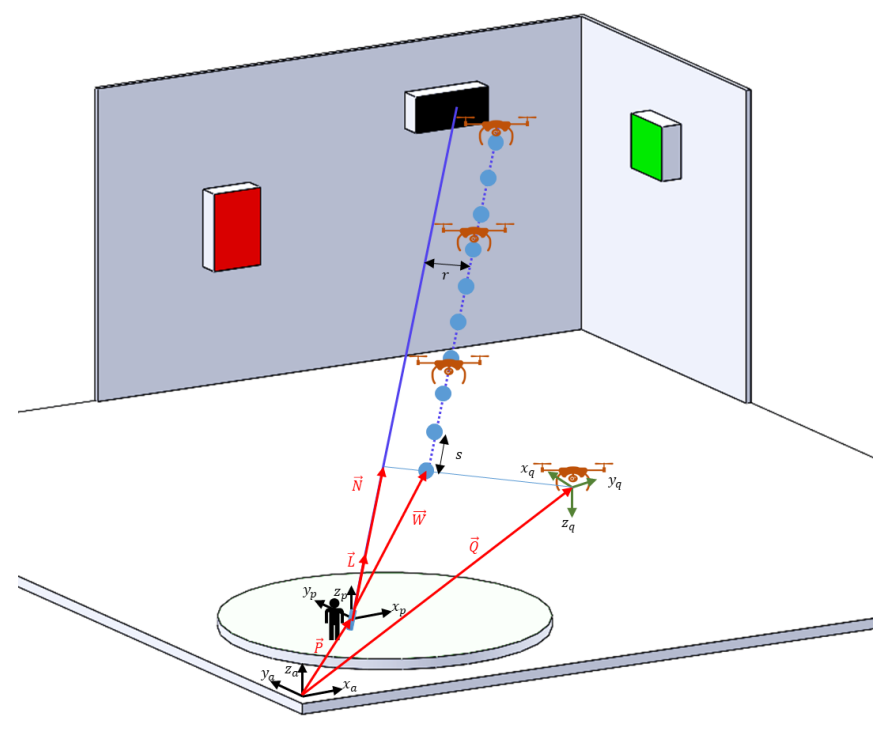

Fig. 4. Illustration of navigation approach showing the operator guiding the UAV using a laser pointer (solid blue line). The dotted blue line is the navigation path with an offset $r$ to avoid laser occlusion. The blue circles represent the waypoints separated with a step size $s$.

\section{System Design}

The system consists of two main subsystems, a pointing handheld device (supervised control device) and a semi-

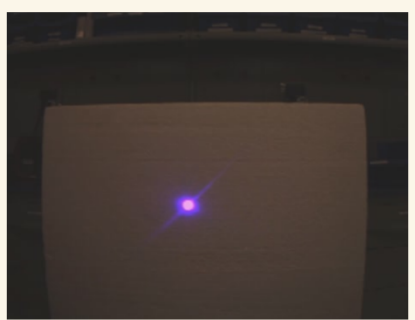

(a) Low Exposure Image

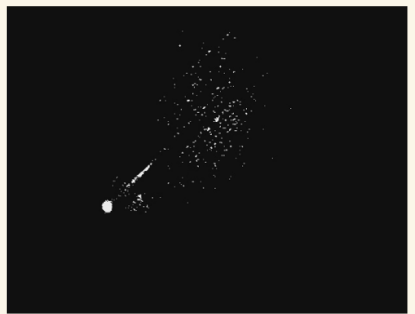

(c) RGB Filter Output

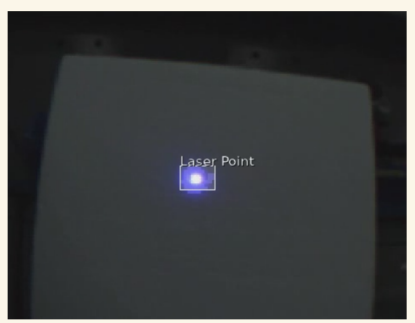

(b) Processed Image

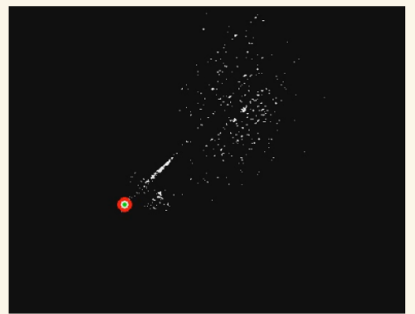

(d) Circle Hough Transform
Fig. 5. Laser light detection using multistage image processing

autonomous UAV system equipped with a programmable flight controller, sensors and a companion computer.

\section{A. Hardware Development}

1) Smart Hand Pointer: Two different designs were considered; the first is based on embedded microcontroller and low-level hardware integration. The second is based on a compact Linux computing module with a higher level of components integration.

Microcontroller Based Pointer: it consists of a 32-bit MCU clocked at $80 \mathrm{MHz}$ and $2 \mathrm{MB}$ memory. Wireless communication is accomplished via a wifi module ESP8266 that is connected serially to the MCU. Orientation angles are measured using a 9-axis bno055 Inertial Measurement Unit (IMU). It is interfaced with the microcontroller using I2C protocol. An embedded circuit for two LED indicators, a digital switch, and a blue laser diode is constructed to complete the system. The system is shown in figure 7.

Linux Stick Pointer: the fundamental difference from the first configuration is employing a compact Linux computer; the Raspberry Pi-zero W running Rasbian Linux OS and the Robotics Operating System (ROS), that is ultra compact, light and has embedded Wifi communication. Inertial measurements and attitude estimation are acquired using a 9-axis IMU device, MYAHRS+, that is connected to the Pi-zero using an I2C bus via its GPIOs. A similar circuit of LEDs, a switch and a laser diode is constructed. These components are directly connected to the computer via embedded GPIOs.

Both approaches are similar in functionality but have some differences in implementation, operation requirements, and integration with the UAV subsystem. Power consumption in the first configuration is a fraction of that of the second configuration, it is around 20-30\%, which is expected given the overhead of running an operating system. On the other hand, due to hardware limitations, the embedded Wifi chip in the first configuration suffers from disconnections 
issues, unlike the RPi-zero integrated wifi antenna that shows more communication reliability especially in a static network configuration for indoor experiments.

2) Quadcopter UAV: The quadcopter frame used is a Storm 4 frame equipped with DJI E310 propulsion system. Low-level flight management is handled by a MindPX controller running PX4 firmware. A companion computer, Odroid XU4 running Linux Ubuntu, is used for high-level control and vision. It also provides the Wifi connection to the pointing device and the ground station. The onboard computer is configured to be the Robotics Operating System (ROS) master in the network that consists of a UAV, a pointing device and a ground station. It is connected to the flight controller serially utilizing the MAVROS package. Two cameras have been considered throughout the development of the system, Pixy CMUcam5 programmable camera and oCam 1CGN-U global shutter board camera. Pixy module provides a standalone image processing with its dual-core processor. While this camera offloads the image processing burden from the companion computer, it offers lower flexibility to use offthe-shelf image processing libraries such as OpenCV unlike our experience with oCam camera.

\section{B. Software Development}

The complete system software is built over ROS Kinetic. ROS network consists of 3 agents, a UAV companion computer (Master), a pointer control device, and a Linux ground station. Communication is accomplished using a static Wifi network. The use of a ground station in this setup is for monitoring and emergency shutdown purposes. Flight control and mode switching logic are embedded on the UAV and pointer computers, see figure 8 . Software running on different physical computing devices are as follows:

- MindPx flight controller: running PX4 firmware that supports Offboard control via MAVLINK. Low level UAV attitude, altitude, and position control are accomplished using tunable cascaded-PID control. It communicates with the ground station (QGroundControl) via MAVROS package and MAVLINK protocol.

- Odroid XU4: configured as ROS master and runs the main offboard control, planning and vision sensing nodes. It receives input data from the pointing device, computes the direction vector, and sends position and heading setpoints to the MindPX flight control unit.

- Handheld Pointer: runs three ROS nodes, the first node is handling the IMU I2C interface and register read. The second node handles necessary data conversion and extraction of physical quantities (raw measurements and Euler angles). The third node manages the laser switching detection and feedback LEDs.

- Ground station: runs system monitoring nodes, camera stream nodes, and emergency control nodes.

\section{Indoor Flight Testing}

The system was tested in the flight arena of the Robotics, Intelligent Systems and Control (RISC) lab at KAUST. The

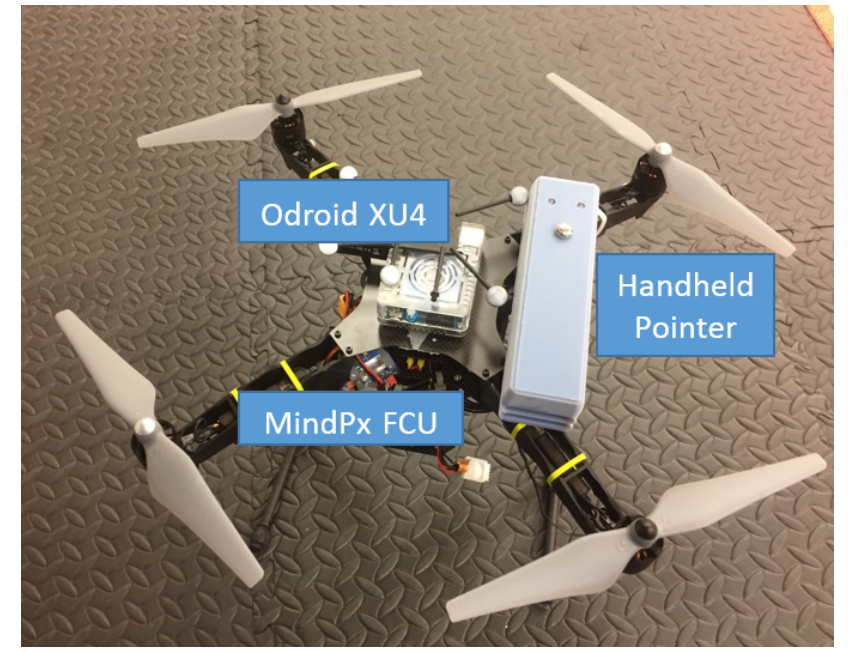

Fig. 6. The Quadcopter system and the handheld pointing device
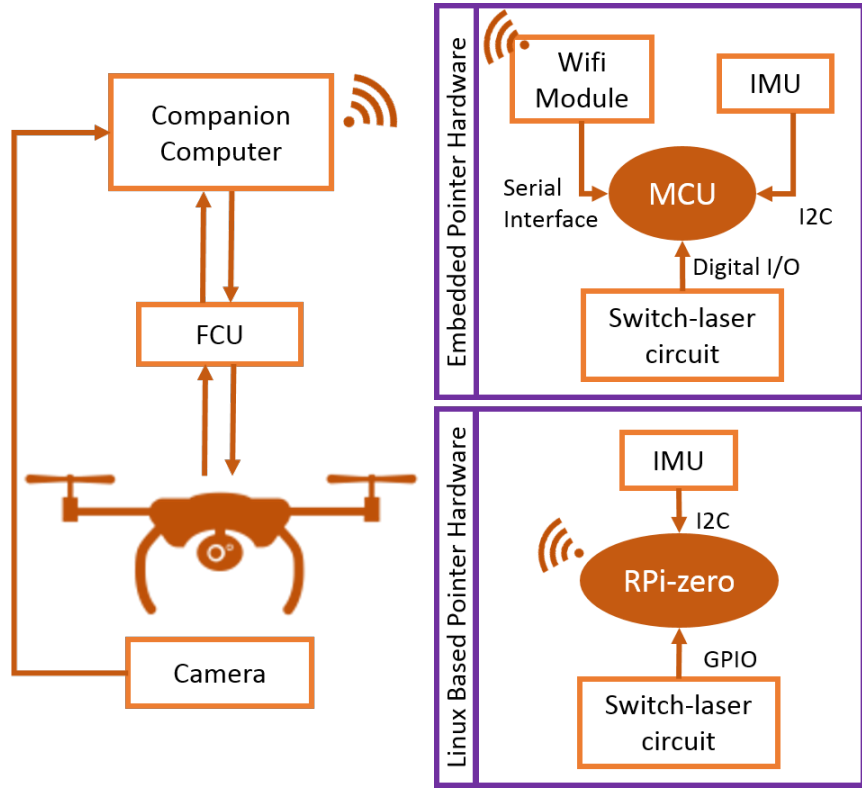

Fig. 7. Pointer controlled UAV hardware system
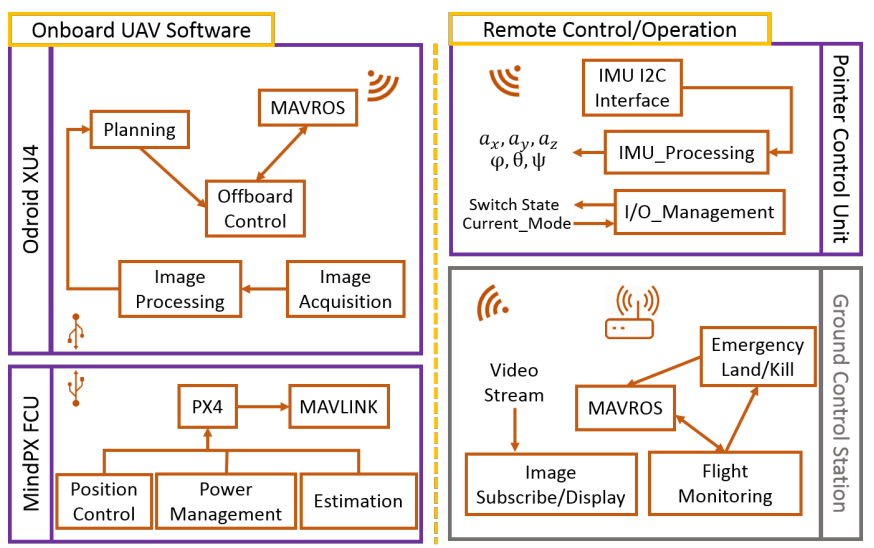

Fig. 8. Software modules running on different devices 
arena is equipped with NaturalPoint OptiTrack motion capture system for accurate indoor positioning. Quadcopter position and attitude data is streamed at $120 \mathrm{~Hz}$ from Motive to the ROS network using Virtual Reality Peripheral Network $(V R P N)$ interface. The flight testing arena is covered by a dedicated dual-band router network where data is streamed over Wifi between the motion capture PC, the ground monitoring station, the handheld pointing device, and the onboard companion computer (Odroid XU4).

Some areas are marked as inspection targets as shown in figure 1 and experiment videos. The pointing device streams its IMU data and digital switch state over Wifi at $20 \mathrm{~Hz}$. The onboard camera can stream images to the ground station at five frames per second over Wifi or store the image data on Odroid memory as a rosbag with other location stamped data.

In this experimental setup, the operator is assumed to be standing in a fixed spot outside the flying arena while holding the pointer. During initialization, the pointer is placed on a flat surface to compute the initial angles offset calculation. The UAV arming and takeoff are manually done using an RC remote controller before switching to offboard control mode to start the autonomous operation. This step can be replaced with a programmed signal from the pointing device or the ground station by calling the corresponding MAVROS services or topics. In offboard mode, control node running on the UAV companion computer receives commands from the pointing device to navigate toward the next target.

Two modes of operation were tested. The first where the UAV returns to the home position and initial heading whenever the laser switch is released. In the second mode, the UAV is commanded to hover in place when the laser switch is not active and returns to the home position only when a return command is explicitly sent. An obstacle sensing node is developed where the cage boundaries are represented as virtual obstacles. This allows the transition from one target to another without going back to the base. Additionally, fast-changing pointing commands were given to the UAV to assess the system's response and real-time adaptation.

\section{CONCLUSION AND FUtURE WORK}

Design, implementation, and testing of a human supervised multirotor aerial robotic system are presented. A Human operator controls the global navigation path of the UAV using a handheld laser pointing device. The onboard vision sensing enables local planning for optimal inspection mission. Indoor flight testing was performed to assess different operating modes of the system. Future directions will consider more advanced path planning and recovery protocols. The visionbased sensing part will be further improved using machine learning methods. For future field experiments, the need for a wireless router can be eliminated through configuring the UAV computer as a wireless server and connect the pointing device directly to this network. More functionality will be added to the device to eliminate the need for additional components needed for UAV arming, takeoff, and landing. Finally, mathematical modeling of the dynamics of human walking is currently investigated to fuse it with IMU measurements to estimate the operator translational motion.

\section{REFERENCES}

[1] M. Quigley, K. Conley, B. P. Gerkey, J. Faust, T. Foote, J. Leibs, R. Wheeler, A. Y. Ng, “ ROS: an open-source Robot Operating System, " In ICRA Workshop on Open Source Software, 2009.

[2] M. Abdelkader, M. Shaqura, C.G. Claudel, W. Gueaieb, “ A UAV based system for real time flash flood monitoring in desert environments using Lagrangian microsensors, "In International Conference on Unmanned Aircraft Systems (ICUAS), (pp. 25-34), 2013.

[3] M. Sudarma, I. Swamardika, and A. Pratama, " Design of quadcopter robot as a disaster environment remote monitor, " International Journal of Electrical and Computer Engineering, (pp. 188-197), 2016.

[4] I. Sa, P. Corke, " Vertical infrastructure inspection using a quadcopter and shared autonomy control, " Yoshida K., Tadokoro S. (eds) Field and Service Robotics. Springer Tracts in Advanced Robotics, vol 92. Springer, 2014.

[5] H. Zakeri, F. Nejad and, A. Fahimifar, " Rahbin: A quadcopter unmanned aerial vehicle based on a systematic image processing approach toward an automated asphalt pavement inspection, " Automation in Construction, 72, (pp.211-235), 2016.

[6] T. Tsan, N. Uchiyama and, O. Sawodny, " Nonlinear controller design for wall inspection by a quad rotor helicopter under wind disturbance, " International Electronics Symposium (IES), (pp. 7-12), IEEE, 2016.

[7] W. Benitez, Y. Bogado, A. Guerrero and, M. Arzamendia, " Development of an UAV prototype for visual inspection of aerial electrical lines, "Seventh Argentine Conference on Embedded Systems (CASE), (pp. 7-12), IEEE, 2016.

[8] W. S. Ng and E. Sharlin, " Collocated interaction with flying robots, "IEEE International Conference on Robot and Human Interactive Communication, (pp. 143-149), 2011.

[9] A. Sanna, F. Lamberti, G. Paravati and, F. Manuri, “ A Kinect-based natural interface for quadrotor control, " Entertainment Computing, (pp. 179-186), 2014.

[10] B. Kim, M. Kim and S. Jo, “ Quadcopter flight control using a lowcost hybrid interface with EEG-based classification and eye tracking, "Computers in biology and medicine, 51, (pp. 82-92), 2014.

[11] S. Adams and C. Friedland, " A survey of unmanned aerial vehicle (UAV) usage for imagery collection in disaster research and management, "9th International Workshop on Remote Sensing for Disaster Response, 2011

[12] C. Eschmann, C. Kuo, C. Kuo and C.Boller, " Unmanned aircraft systems for remote building inspection and monitoring, " Proceedings of the 6th European Workshop on Structural Health Monitoring, 2012.

[13] A.R. Jimenez, F. Seco, C. Prieto and J. Guevara, " A comparison of pedestrian dead-reckoning algorithms using a low-cost MEMS IMU, " In IEEE International Symposium on Intelligent Signal Processing (pp. 37-42), 2009.

[14] J.G. Park, A. Patel, D. Curtis, S. Teller and J. Ledlie, “ Online pose classification and walking speed estimation using handheld devices," In Proceedings of the ACM Conference on Ubiquitous Computing (pp. 113-122), 2012.

[15] D.H. Ballard, " Generalizing the Hough transform to detect arbitrary shapes, " In Readings in computer vision (pp. 714-725), 1987.

[16] I.E. Paromtchik, " Optical guidance method for robots capable of vision and communication, "In Robotics and Autonomous Systems 54 (pp. 461-471), 2006. 